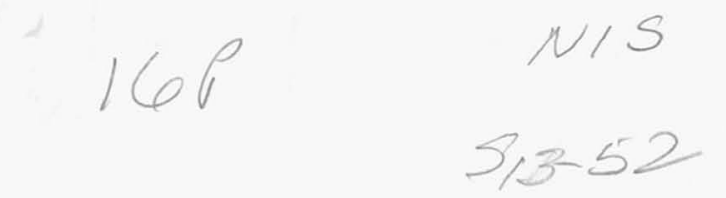

N9 $91-2558 \frac{446 / 1}{P}, 16$

\title{
A Systems Approach to the Physiology of Weightlessness
}

\author{
Ronald J. White, * Joel I. Leonard, John A. Rummel, \\ and Carolyn S. Leach
}

\begin{abstract}
This paper presents a systems approach to the unraveling of the complex response pattern of the human subjected to the challenge of weightlessness. The major goal of this research is to obtain an understanding of the role that each of the major components of the human system plays following the transition to and from space. The cornerstone of this approach is the utilization of a variety of mathematical models in order to pose and test alternative hypotheses concerned with the adaptation process. An integrated hypothesis for the human physiological response to weightlessness is developed.
\end{abstract}

\section{INTRODUCTION}

Research concerned with the physiological adaptation of man to the weightless environment of space is replete with special challenges. In the past, manned space flights have been relatively infrequent and expensive, with the result that only a small number of subjects have been available and the statistical significance of the observed changes has been difficult to assess. In addition, for valid and understandable reasons, the types of measurements made on man have been limited. Equipment used in the environment of space has had to be specially designed for each task, and the development and testing of major equipment has required long lead times. Finally, any observed physiological response in the normal astronaut population is actually the result of a combination or integration of the responses of several different body systems, each responding simultaneously to the challenges of space flight and weightlessness. The individual systems involved are themselves complex, containing both competing and redundant pathways and multiple interconnections.

From the Management and Technical Services Company, Houston, Texas and Johnson Space Center, National Aeronautics and Space Administration, Houston, Texas.

This paper was presented at the 15th Hawaii International Conference on System Sciences, Honolulu, Hawaii, January 1982, and is republished here with the permission of HICSS.

*Address all correspondence to R. J. White, MATSCO, 1050 Bay Area Boulevard, Houston, Texas 77058.

343

0148-5598/82/0800-0343\$03.00/0 @ 1982 Plenum Publishing Corporation 
In the future, some of these problems related to space flight are likely to disappear. In the new Shuttle era, manned flights, in near Earth orbit, will be frequent, and the number of astronauts going into space will be considerably larger than in any previous space program. A large number of special life sciences experiments utilizing the Spacelab module will be conducted, and the diversity of the physiological measurements made will be greater than ever before. In preparation for the future flight opportunities, an inventory of special flight-tested life sciences laboratory equipment (LSLE) is currently being built. In effect, the ground rules are changing so that life sciences research in general, and human physiological research in particular, are becoming easier to conduct in the weightless environment of space.

This paper is concerned with the most difficult problem remaining in the study of man's adaptation to weightlessness and subsequent readaptation to the environment of Earth. This problem involves unraveling the integrated total human physiological response to obtain an understanding of the role that each of the major components of that system plays following the transition to and from space. In its basic form, this problem is not a new one in physiology or medicine. A similar problem is encountered whenever one subjects a complex system to a stress that affects several components of the system at once. This paper will present a general systems approach to conducting and analyzing research on the human adaptation to weightlessness. The cornerstone of this approach is the utilization of a variety of mathematical models in order to pose and test alternative hypotheses concerned with the adaptation process. This general approach will be illustrated by considering certain aspects of the problem of fluid and electrolyte shifts in weightlessness. Finally, an integrated hypothesis based on numerous simulation studies and available experimental data will be presented.

\section{MODELING}

Each of us has, through experience, built up a sense of what a model is. In effect, it is an image of reality that captures the essence of some aspect of form and function from something else. In the sciences, one's notions about a particular system often can be represented in symbolic terms, with real quantities and processes replaced by symbols and mathematical operations. The result of this representation is an axiom system that can be identified with what is called a mathematical model. Such a model is usually an imperfect image of reality, except in trivial cases. The discovery of the imperfections of a model can be time-consuming and fraught with difficulty because of the fact that most mathematical models of interest in the life sciences are nonlinear and involve coupled differential equations. In that case, heavy computational machinery is usually involved in extracting some of the consequences of the axiom system. This ties the use and development of major models in the life sciences to the more powerful computing systems. However, many of the simpler models can be studied effectively using the new generation of microcomputers, ${ }^{1}$ and this advance should do much to popularize the use of such models, especially as teaching aids.

In science, mathematical models are built and studied for several reasons. The very act of constructing a model requires systematic, logical thinking, and generally provides new insight into the organization of the system elements, the processes within 
the elements themselves, and the multiple pathways connecting the system elements. Model building leads almost immediately to the identification of gaps in the experimental knowledge of the system of interest and suggests particular ways of filling those gaps. In addition, models can provide information concerning the regularities and patterns of behavior that a system possesses. When a system is complex, such patterns can be hidden from view simply because one does not know what to look for. By clarifying what to look for, a model may predict both correlations and cause-and-effect relations that are subject to actual verification in the real system.

If one considers a model to be a collection, $\mathrm{H}$, of hypotheses, then $\mathrm{H}$ is said to imply $\mathrm{C}$, where $\mathrm{C}$ represents the collection of conclusions of the model. By observing $\mathrm{C}$, or part of $\mathrm{C}$, in the real system, one would like to turn things around and say that $\mathrm{C}$ implies $\mathrm{H}$. In general, this is impossible, but by matching part of the predictions with actual data, one generates a level of confidence in the correctness of the hypotheses. This level varies with the size of the set $C$ that is matched, and it is difficult to quantify. In practice, the most useful results are obtained when the observed $\mathrm{C}$ does not match the predicted $\mathrm{C}$ exactly. Analysis of the differences involved can then lead to a modification of the hypotheses that define the model, or to the need for new and clarifying experiments. Such a process is generally iterative, being applied over and over again with new stresses designed to force the model to display more and more of its weaknesses. Reconstruction of the model by modification of the hypothesis set leads to a stronger model, in some sense. As the model behavior moves closer to the behavior of the real system through iterative modification of the hypotheses, the probe of science sinks ever deeper into the subject. In fact, this iterative use of models is only a restricted form of the general scientific method. In the next section, this approach is formalized and specialized for the study of the physiological problems of weightlessness.

Each of the particular models used in this project is a deterministic, nonlinear representation of a major human physiological system. All models are implemented on a digital computer system using a finite difference formulation, and all are used in an interactive time-sharing mode with automated graphical output. The models, taken together, are able to provide simulations of a wide variety of physiological events spanning a large time frame, from seconds or minutes to months in length. The basic models in use are a pulsatile cardiovascular model, ${ }^{2,3}$ a respiratory model, $, 4,5$ a thermoregulatory model,,${ }^{6,7}$ an erythropoiesis regulatory model, ${ }^{8}$ a circulatory, fluid, and electrolyte balance model,, 910 and, a model of calcium regulation. ${ }^{11}$ In addition, the first five of these models have been merged into a common framework termed the "whole-body algorithm, ${ }^{, 12}$ which has been used to simulate composite human function, particularly during space flight. The six basic models are illustrated conceptually in Figures 1 and 2, and the whole-body algorithm is shown in Figure 3. All models are characterized by an active controlling system that regulates a relatively passive controlled system, and, taken together, these two major components function as a (usually negative) feedback control system. The major feedback variables present in these models represent many of the actual sensors present in the body, including temperature sensors, chemoreceptors, baroreceptors, oxygen sensors, and osmoreceptors. The models were validated using a wide variety of experimental and clinical conditions, and a partial list of these validation studies is presented in Table 1. With all of these 

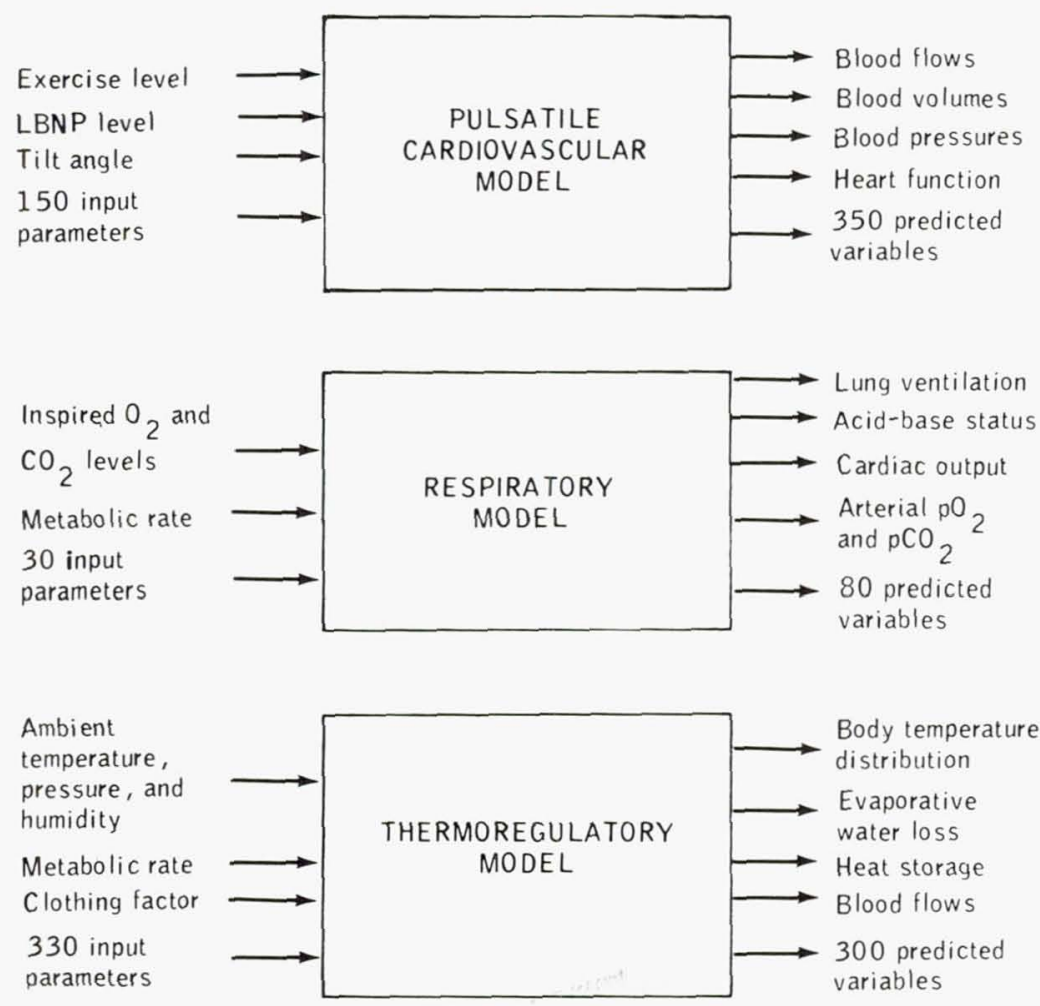

Figure 1. Models used for simulating short-term physiological events.

models, multiple stresses and sequential stresses of varying degrees can be applied, just as in an actual experimental protocol. A few typical simulations produced by some of these models will be discussed later in this paper.

Table 1. Stresses Related to Space Flight That Were Used to Validate the Responses of the Simulation Models

\begin{tabular}{lc}
\hline Hypogravic stress & Environmental disturbances \\
Supine bed rest & Hypoxia \\
Head-down bed rest & Hypercapnia \\
Water immersion & Temperature \\
& Ambient pressure \\
Orthostatic stress & Fluid shifts \\
LBNP & Hemorrhage \\
Tilt table & Infusion \\
Postural change & Water and salt loading \\
& Dehydration \\
& Metabolic stress \\
& Exercise \\
& Diet restriction \\
\hline
\end{tabular}



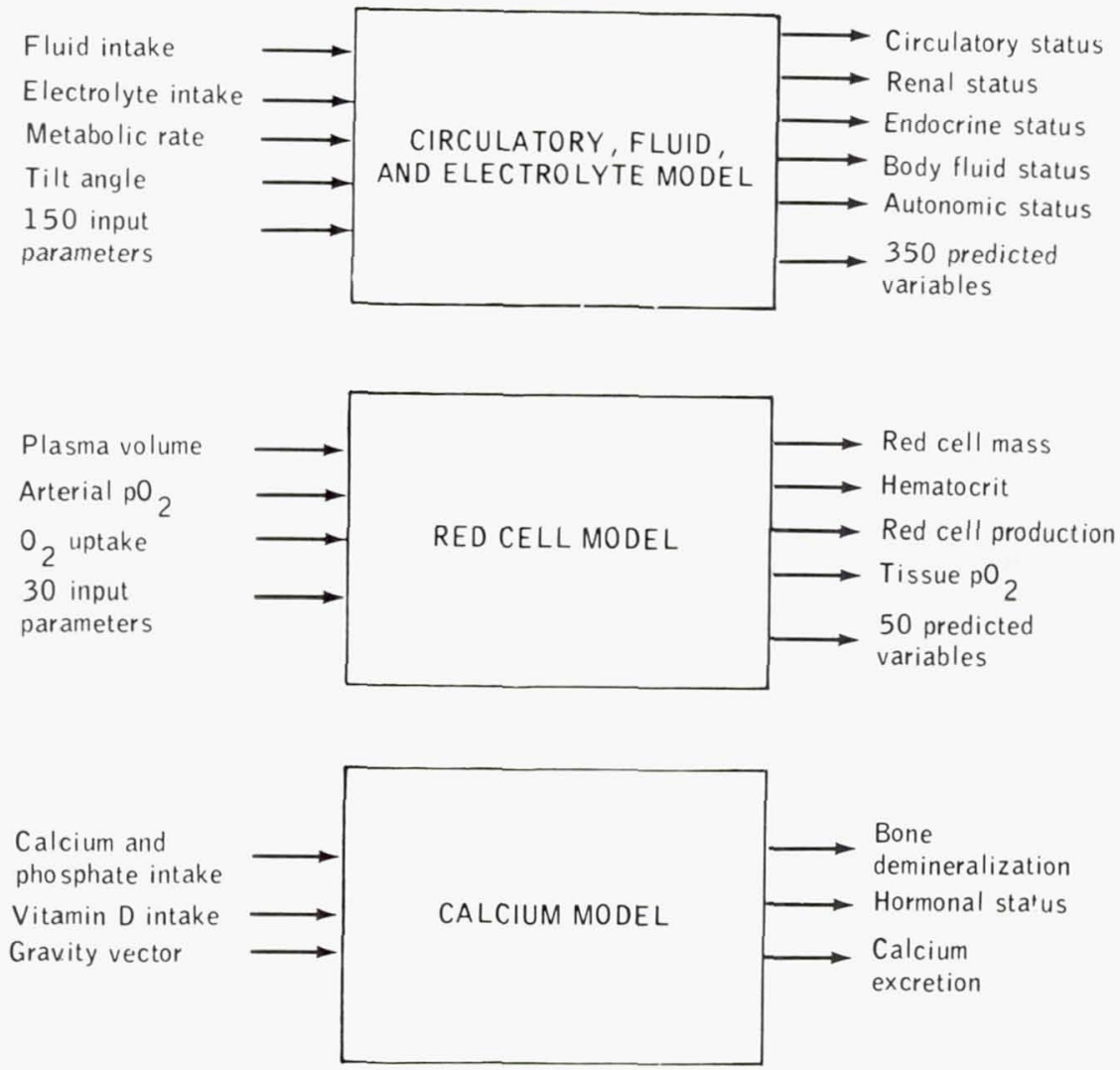

Figure 2. Models used for simulating long-term physiological events.

\section{SYSTEMS ANALYSIS}

Figure 4 presents the general systems approach designed for the study of the physiology of weightlessness. This restricted, but powerful, form of the scientific method has been implemented using the specially designed integrated medical data analysis system shown in Figure 5. The systems analysis approach is clearly iterative, and one may enter the main loop at any point. The automated data base and analysis system permits large arrays of space-flight and pertinent ground-based data to be scanned rapidly, while variables are correlated visually or automatically, and hypotheses are tested for statistical significance. Such an evaluation of experimental data can lead, under appropriate conditions, to a qualitative formulation of alternative mechanisms involved in producing the observed responses.

The next step, which requires an in-depth understanding of the capabilities and limitations of the relevant model or models in physiological terms, draws heavily on the theory of feedback-regulating systems and requires that the hypotheses in question be formulated in quantitative terms suitable for actual testing using one or more of the models available. The integrated medical data analysis system (Figure 5) was designed 


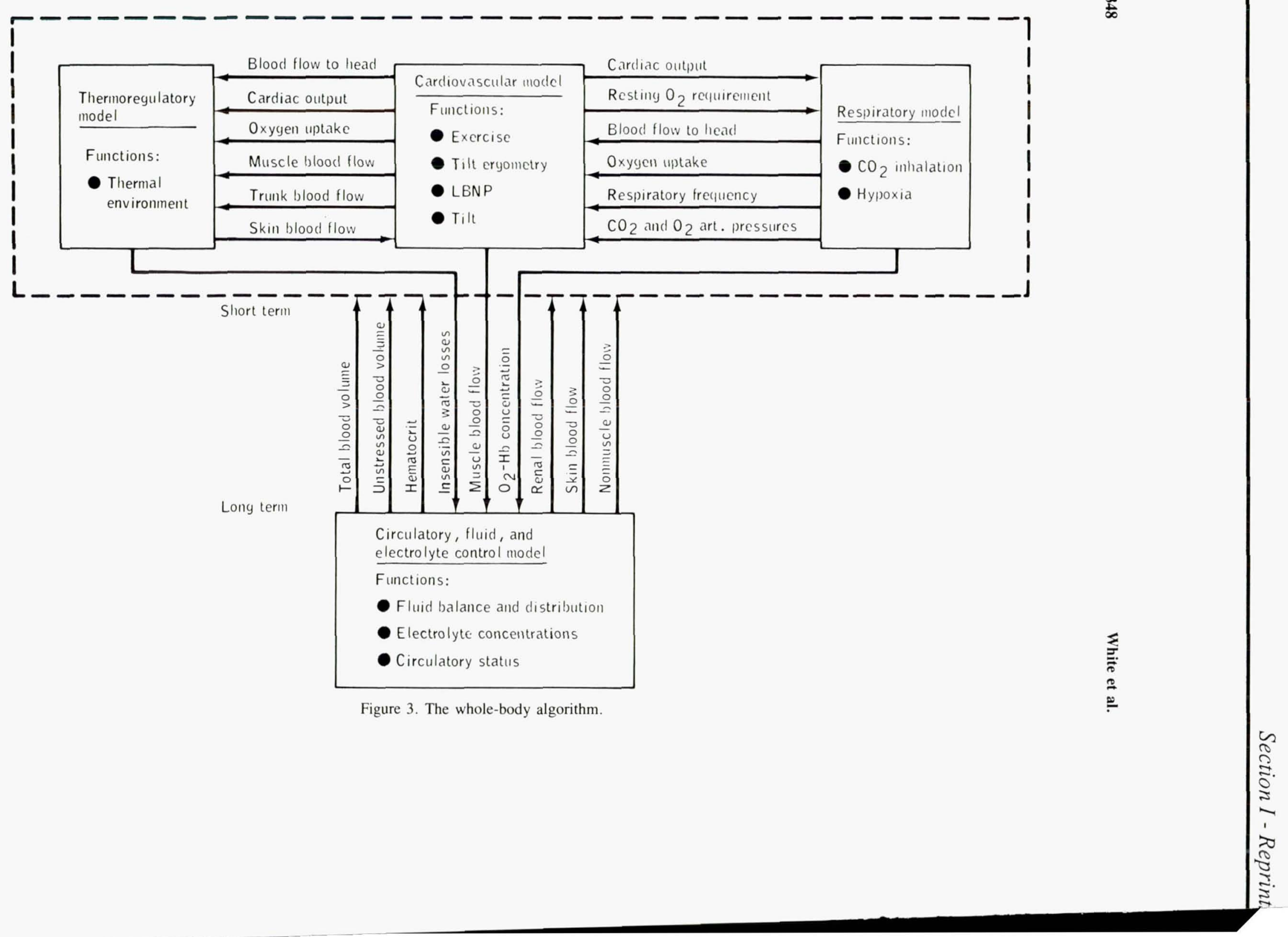




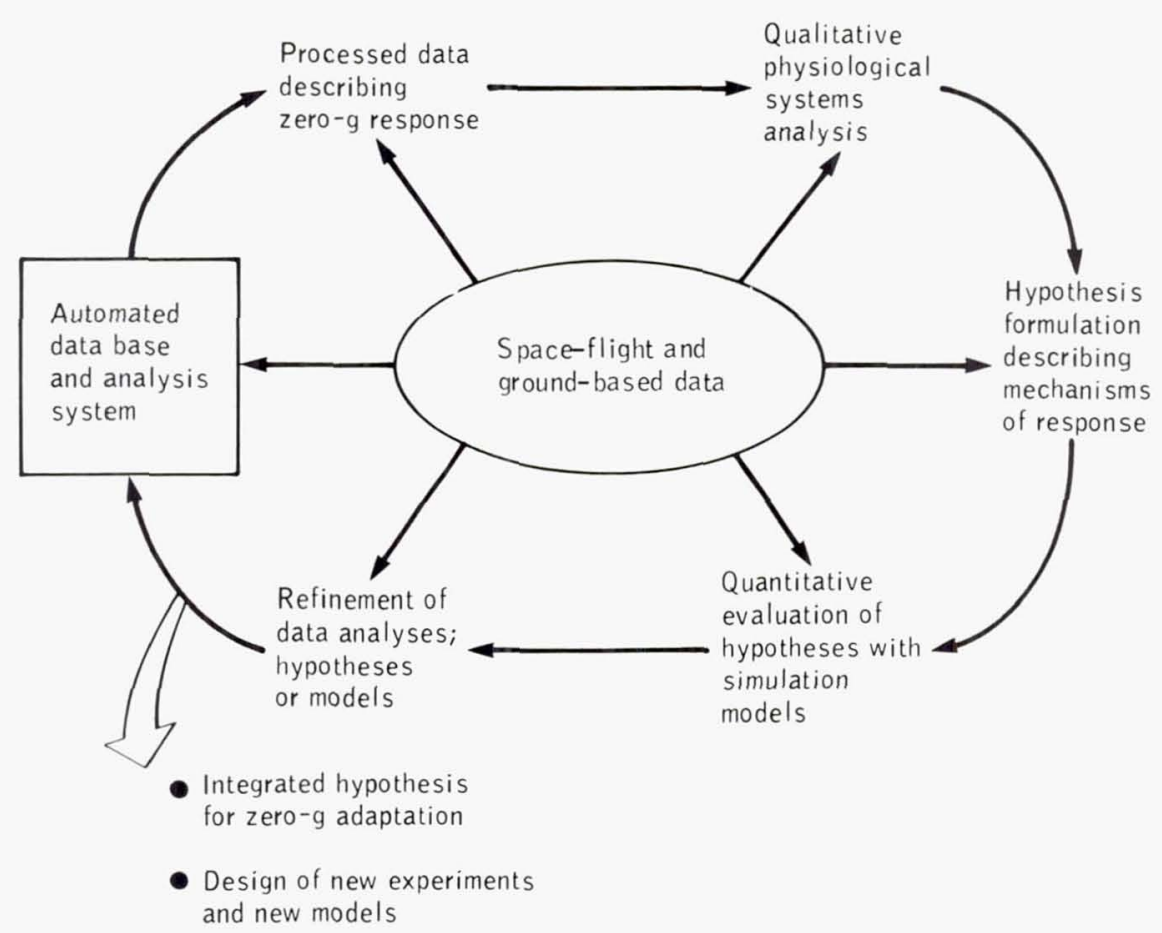

Figure 4. Systems analysis approach to understanding the physiology of weightlessness.

so that the elements could interact in either sequential or parallel fashion, enabling a portion of the actual experimental data to be employed as input (forcing) functions to a simulation model while the model output can be compared to another portion of the experimental data in the data base.

Actual testing of the hypotheses using the available models constitutes the next step in this process. Good agreement between model output and experimental data furnishes a certain level of confirmation for the hypothesis under consideration but does not "prove" that the hypothesis is true. As in almost all applications of the scientific method, there are only varying degrees of confirmation involved in the process, not absolute certainty. Poor agreement or contradictions between model output and actual data cause one to question the hypothesis under investigation, the structure of the model itself, or the validity of the experimental data. In actual practice, such a state of poor agreement, at least initially, is the more interesting one, and leads to a level of improvement in one of the above major elements. In this way, the modeling process contributes to an increased understanding of the system.

This whole process must be able to be applied consistently to man in space or on Earth during various maneuvers, since the same physiological systems are involved in both cases, at least initially. Some Earth-based studies are particularly relevant to the function of the body during weightlessness, and they have been used as experimental analogues of weightless space flight. These include water immersion, bed rest, and 


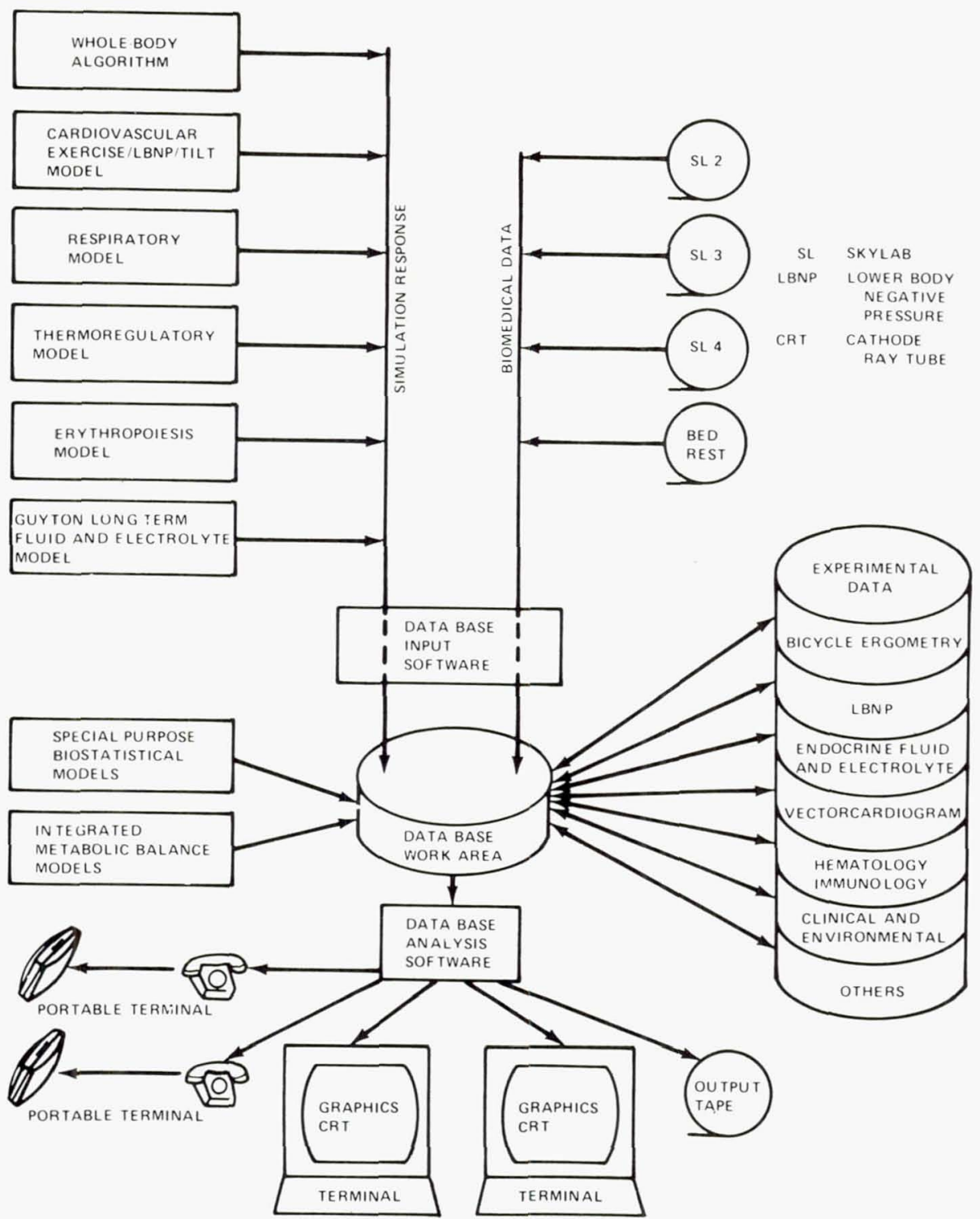

Figure 5. Integrated medical data analysis system.

head-down bed rest studies, and the modeling of such studies has been an important part of our overall analysis program.

\section{RESULTS}

A large number of simulations of a variety of types using all of the models mentioned earlier in this paper have been completed. Some of them have been reported 
on previously ${ }^{2-17}$ and a book, which contains others, is currently in preparation. Reporting on even a fraction of these actual studies is outside the scope of this paper, and only a sample of this work is presented here. At the end of this section, an integrated hypothesis, combining the final result of many of the individual studies, will be discussed.

Responses of the body systems that regulate human fluid and electrolyte balance to the stress of weightlessness can be divided into at least two distinct phases. The acute phase is characterized initially by a reduction in the normal gravity-induced hydrostatic gradients and a rapid headward shift of fluid. Then, the body rapidly responds, through feedback, to relieve some of the stresses felt by the central portion of the circulation. This acute phase lasts for 1 to 2 days and is followed by an adaptive phase, which is characterized by much less dramatic change. As mentioned in the previous section, ground-based analogues of true weightlessness are particularly useful because of the limited information gathered on previous space flights and because of the fact that the same physiological systems are involved in the responses to the similar stresses. A mathematical model provides a framework to systematically examine the various hypogravic stresses and to study their similarities and differences. Simulations are presented of two of these analogues of true weightlessness in order to illustrate typical model capabilities.

Figure 6 presents the results of a simulation study of $5^{\circ}$ head-down tilt in man using a modified version of the model of Guyton. ${ }^{9}$ This modified model has leg vascular and tissue compartments, gravity-dependent circulatory elements, and a natriuretic factor. At the break in the curves shown in Figure $6(t=0)$, the angle of body tilt (to the horizontal) was modified and the elastic forces in the tissues and vessels themselves redistributed the body fluids in a manner favoring upper-body hypervolemia. Ordinate scales are omitted in this figure to emphasize the qualitative aspects of changes pictured. Quantitative data are presented in Table 2. Subsequently, feedback controllers respond by regulating blood flow, blood pressure, and central blood volume toward more normal values. Autonomic, renal, and hormonal control elements all contribute significantly in this response. Table 2 compares the results of this simulation study with the actual data recently collected after 24 hours of such tilt by Blomqvist. ${ }^{18}$ Examination of this table and comparison of Figure 6 with an idealized figure presented by Blomqvist (his Figure 2) shows that the simulation presents a qualitatively and quantitatively accurate (for most Figure 6 variables, given normal variability) picture of the events involved in head-down tilt. The usefulness of this simulation will be addressed later when many such studies are integrated into a discussion of space flight.

Figure 7 presents a simulation study of water immersion in man. Here, fluid was shifted out of the lower part of the body by the application of external compression on the leg interstitial and venous compartments. The results of this maneuver are similar to, but not identical with, the head-down tilt study shown in Figure 6. Comparison of the water immersion simulation to average experimental data ${ }^{19,20}$ shows that the simulation results are representative of the typical response.

By comparing the results of simulation studies of head-down tilt, water immersion, lower body positive pressure, supine bed rest, and space flight, it is possible to determine the pathways and mechanisms in the model that play a determining role in 

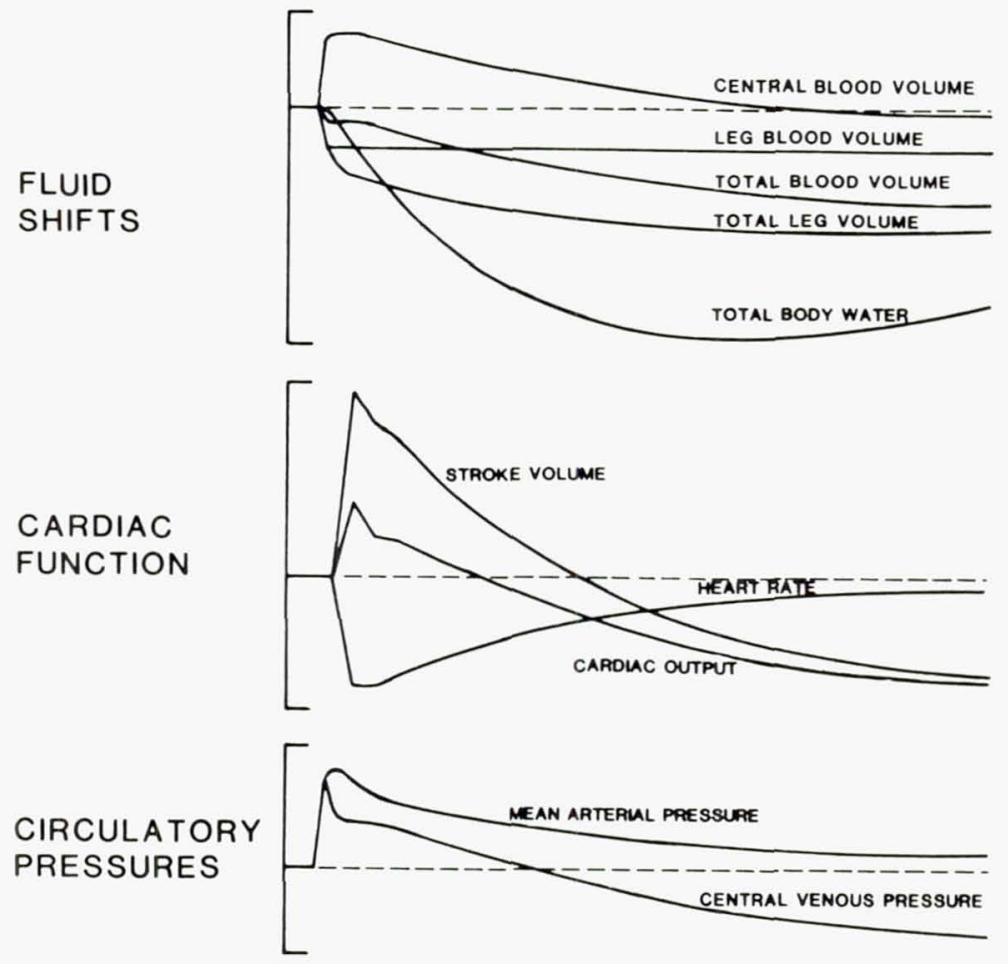

RENAL FUNCTION
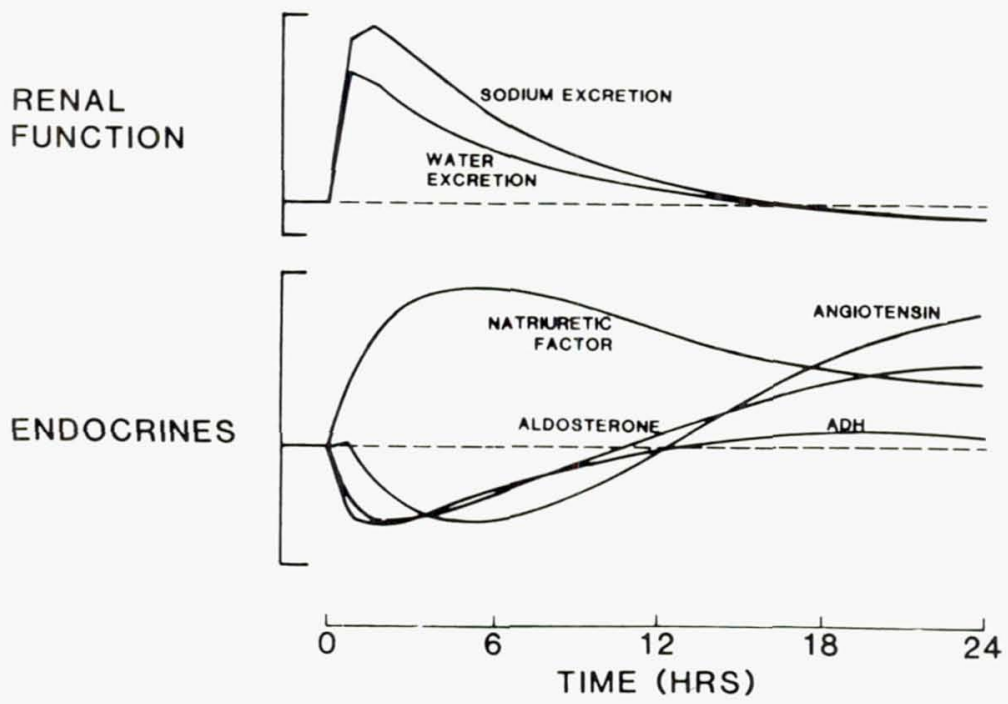

Figure 6. Simulation of head-down tilt.

each of these related stresses, and to suggest key experiments that can confirm these pathways and mechanisms in man. This has been done in the case of space-flight studies and has led to the proposal of several possible future Shuttle experiments. A major study of the acute aspects of fluid shifts during weightlessness will take place on 
Table 2. Comparison of Model Output and Experimental Response for $24-H o u r$ Head-Down $\left(-5^{\circ}\right)$ Tilt Study

\begin{tabular}{lcc}
\hline & \multicolumn{2}{c}{ Value at hours compared to control } \\
\cline { 2 - 3 } \multicolumn{1}{c}{ Quantity } & Experiment ${ }^{18}$ & Model \\
\hline Fluid shifts & & \\
Total body water & $-1300 \mathrm{ml}$ & $-1130 \mathrm{ml}$ \\
Leg blood volume & - & $-256 \mathrm{ml}$ \\
Leg interstitial volume & - & $-454 \mathrm{ml}$ \\
Leg volume & $-900 \mathrm{ml}$ & $-710 \mathrm{ml}$ \\
Total blood volume & $-425 \mathrm{ml}$ & $-563 \mathrm{ml}$ \\
Urine rate, 8 hr/24 hr & $127 \%$ & $190 \%$ \\
Hemodynamics & & \\
Cardiac output & $-7.8 \%$ & $-11.1 \%$ \\
Stroke volume & $-8.5 \%$ & $-9.8 \%$ \\
Heart rate & $0 \%$ & $1.4 \%$ \\
Arterial pressure & $+4 \%$ & $+3 \%$ \\
Central venous pressure & $-49 \%$ & $-9 \%$ \\
Left atrial pressure & - & $-50 \%$ \\
Hormones & & \\
Aldosterone & $+35 \%$ & $+17 \%$ \\
Angiotensin & $+25 \%$ & $+27 \%$ \\
ADH & $+57 \%$ & $+13 \%$ \\
Natriuretic factor & - & \\
\hline
\end{tabular}

the dedicated life sciences mission to be conducted by the United States in 1985.

Figure 8 presents an integrated hypothesis for the human physiological response to weightlessness. This hypothesis includes most of the major systems of the body. It was generated from the systems approach discussed earlier (Figure 4) through simulation studies using the models already mentioned. The experimental data, without which these modeling studies would have been impossible, are reported in several places. ${ }^{21-24}$ Although a great amount of detail has been omitted from Figure 8 for the sake of clarity and simplicity, the broad interactions among many of the body's systems are presented in a unified fashion. The following picture has emerged. Disturbances in the cardiovascular, fluid-electrolyte, erythropoietic, musculoskeletal, and metabolic systems, which are found during and after space flights of varying duration, appear to be attributed to two major effects of weightlessness. These are, first, the absence of hydrostatic forces, resulting in severe fluid shifts within the body, and second, the absence of deformation forces, resulting in degradation of normally loadbearing tissues. The first of these effects leads to a reduction in body fluids - most important, blood volume. The major consequence of the second effect is a reduction in bone and muscle mass. In addition, a third factor, a long-term alteration of metabolic state, which itself reflects changes in dietary intake and exercise, was found to play an important role in the responses of the Skylab crews. All of these events have both acute and long-term effects that lead to the notable and consistent findings of loss in weight, change in body composition, decreased tolerance for orthostasis, and, upon return to a $1-\mathrm{g}$ environment, a compromised response to physical activity. Adaptation is said to occur when the body adjusts to these changes and reaches a new steady-state level. 


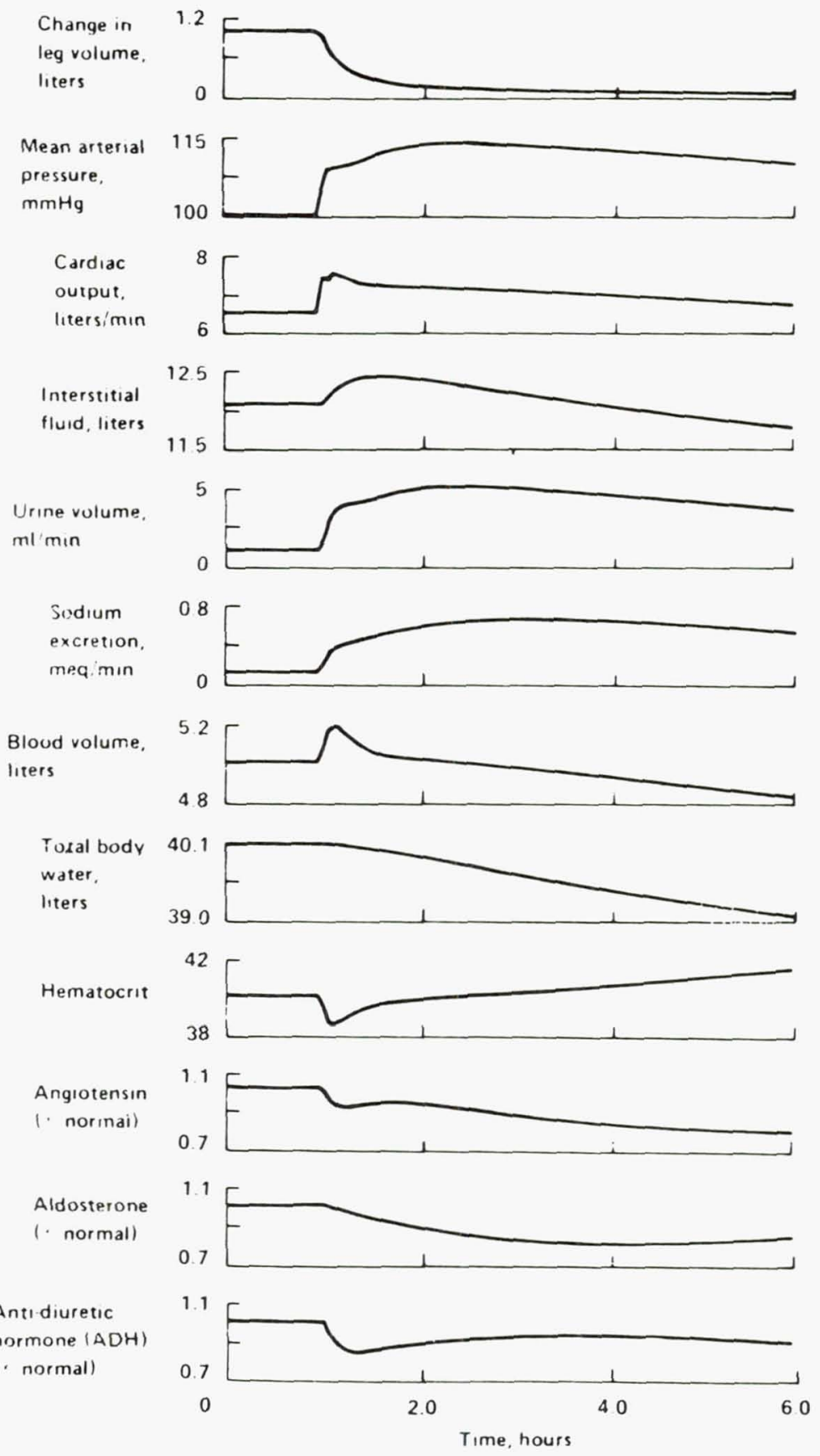

Figure 7. Simulation of water immersion 


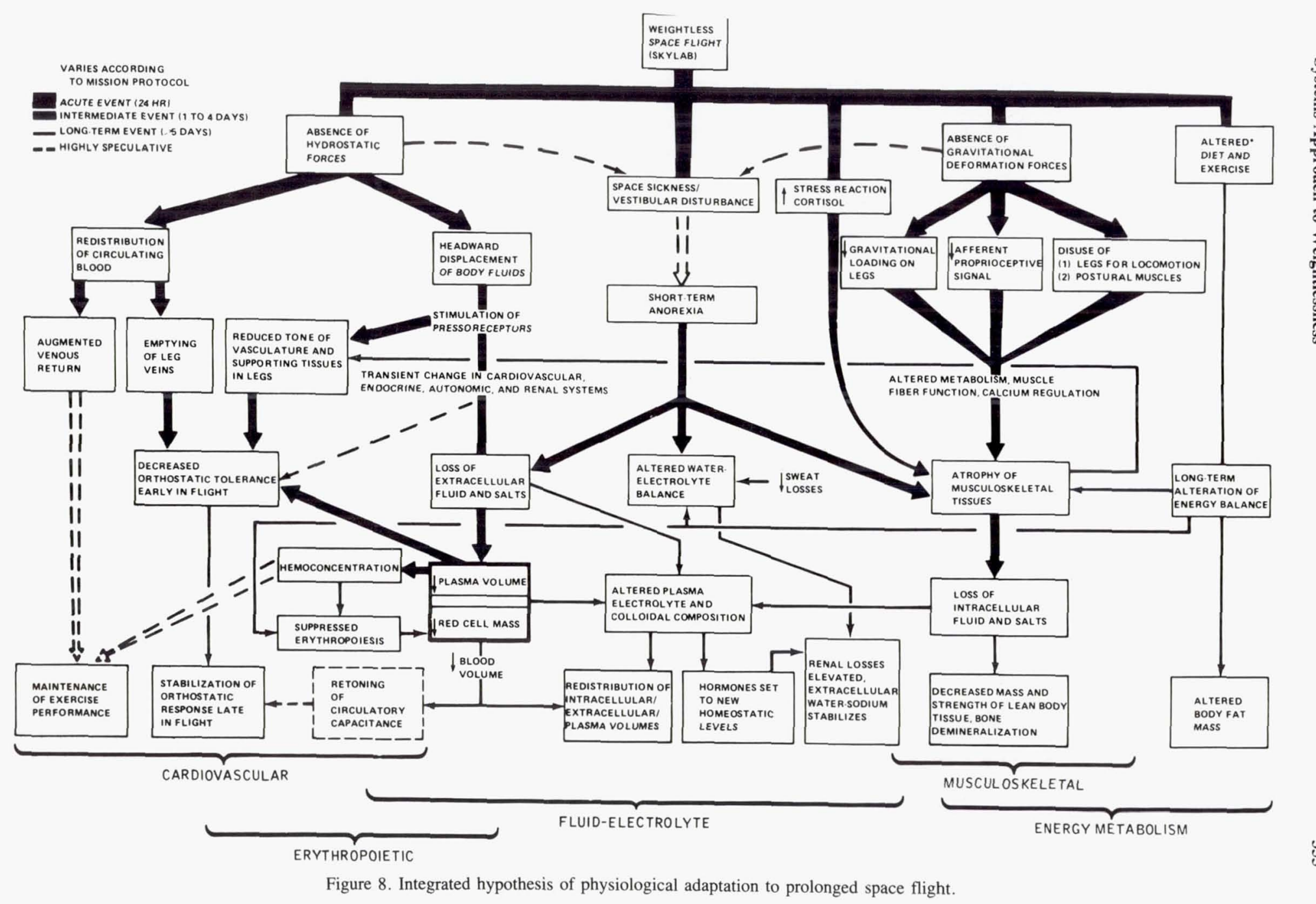


Figure 9 is an attempt to show the relative time course of adaptation for each major physiological system. The return to baseline reflects the establishment of a new homeostatic level appropriate to weightlessness.

Our simulation studies have supported the concept that, within the time span that man has so far been studied in space, these responses to weightlessness can be explained in terms of normal feedback regulatory processes. One classical example of such processes concerns the blood volume controllers that reduce plasma volume when challenged by the cephalic shifts of peripheral fluid. Also, the reduction observed in red cell mass has been postulated to be partly a result of the homeostatic response to hemoconcentration and tissue hyperoxia. Another of many examples concerns the biochemical mechanisms that sensitively respond to small changes in electrolyte shifts resulting from cell demineralization, and, within limits, maintain the plasma composition at the expense of excess renal excretion.

Exposure to weightlessness invariably leads to losses of major body constituents at rates that, according to the present analysis, are disproportionate to their concentrations in the body. The most rapid losses are observed for extracellular fluids and salts, and are reflected by equally rapid decrements in leg volume. At the other exteme is a class of substances that are lost by the body much more slowly. Calcium and perhaps red cells are representative of this category. Depending on the degree to which caloric intake matches energy requirements, fat stores can be included in this group as well. Muscle tissue appears to degrade at an intermediate rate, as exemplified by nitrogen and potassium losses. All these rates of disappearance from the body most likely depend on the nature of the disturbance, and on the effective time constant of the correcting homeostatic system.

Our simulation studies have confirmed the hypothesis previously suggested that the loss of blood volume is of central importance to the understanding of the zero-g responses of several major systems. While this loss is believed to be an acute circula-

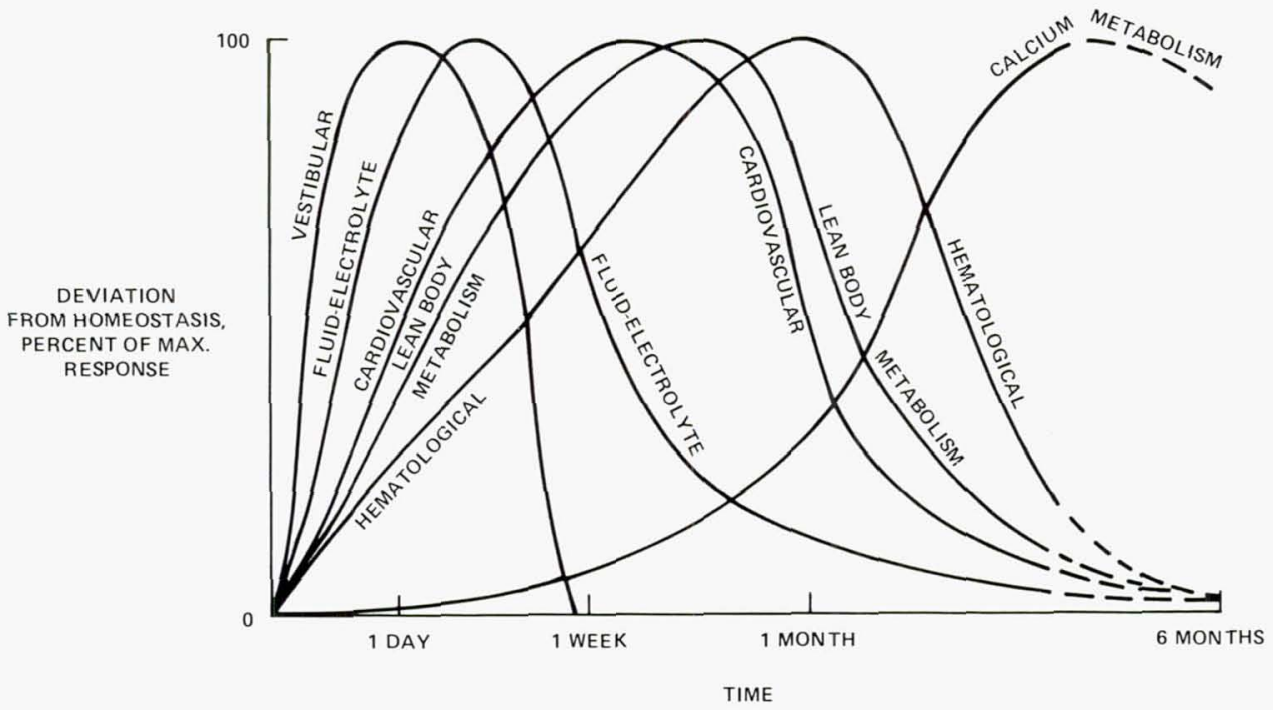

Figure 9. Approach toward homeostasis of physiological systems during space flight. 
tory adaptation to volume overload, it was found to play a significant long-term role in the orthostatic intolerance and reduced exercise performance observed postflight. Also, the reduced plasma volume was found to have a potentially strong influence on the erythropoietic response. Finally, the long-term adaptation of the circulation may depend on the vascular elements responding to accommodate the hypovolemic condition. It should be noted, however, that in none of these cases was the loss of blood volume alone sufficient to explain the entire response. Another event that had a widespread effect was the negative energy balance noted for the crews of the two shortest Skylab missions and for all crews during the early flight period. In addition to the obvious effect on fat stores, an inadequate intake of fluid and food was found to be implicated in the lack of expected acute renal response, the loss of muscle tissue, the loss of water, and the differential loss of red cells among the crews.

\section{CONCLUSION}

The contributions of the systems analysis approach, in general, and the mathematical models, in particular, were invaluable in constructing the hypothesis discussed above. This hypothesis should be treated with appropriate caution, since some of the interconnections between the elements shown in Figure 8 are still tentative and have not been confirmed by direct experimental evidence. In spite of this limitation, these studies have led to an improved understanding of the physiology of weightlessness. When this understanding is even more complete, it should be possible to define appropriate indices of health (normal adaptation) during space flight and, using models, to predict individual responses to weightlessness. Combining these two elements, it should be possible to develop appropriate counter measures to the deleterious effects of space flight (upon return to Earth).

There are many additional benefits derived directly and indirectly through the systems approach using mathematical modeling, as sketched in this paper. For example, the models themselves serve as composite references to the present state of knowledge of the different physiological systems. As new information is obtained about the function or control of a particular system, that information can be used to improve or update the model in question. Thus, a model implicitly can contain the theoretical strengths and weaknesses of an entire field of study. Use of such knowledge can be an effective means of guiding or planning a research program, and one of the spinoffs of modeling and the systems approach concerns the area of research planning. A second additional benefit, only alluded to earlier, concerns the potential applicability of these same models to the study of abnormal states and, therefore, to clinical problems. At present, this application is one of the major challenges of the future.

\section{ACKNOWLEDGMENTS}

The authors gratefully acknowledge the support of the National Aeronautics and Space Administration through contracts NAS 9-15487 and NAS 9-16328. One of us (R.J.W.) benefited greatly from numerous discussions with Arthur C. Guyton on the uses of modeling in physiology and medicine. 


\section{REEERENCES, \\ i 6 \& 4 is}

1. Randall, J. E. Microcomputers and Physiological Simulation, Addison-Wesley, Reading, Mass., 1980.

2. Croston, R. C., Rummel, J. A., and Kay, F. J. Computer model of cardiovascular control system response to exercise. J. Dyn. Syst. Meas. Control 95:301-307, 1973.

3. Croston, R. C., and Fitzjerrell, D. G. Cardiovascular model for the simulation of exercise, lower body negative pressure, and tilt experiments. Modeling and Simulation 5:471-476, 1974.

4. Grodins, F. S., Buell, J., and Bart, A. J. Mathematical analysis and digital simulation of the respiratory control system. J. Appl. Physiol. 22:260-276, 1967.

5. Gallagher, R. R. Investigations of Respiratory Control Systems, NASA CR-160213, 1973.

6. Stolwijk, J. A. J. A Mathematical Model of Physiological Temperature Regulation in Man, NASA CR1855,1971

7. Leonard, J. I. Modifications to the Steady-State 41-Node Thermoregulatory Model Including Validation of the Respiratory and Diffusional Water Loss Equations, NASA CR-160219, 1974.

8. Leonard, J. I., Kimzey, S. L., and Dunn, C. D. R. Dynamic regulation of erythropoiesis: A computer model of general applicability. Exp. Hematol. 9:355-378, 1981.

9. Guyton, A. C., Coleman, T. G., and Granger, H. J. Circulation: Overall regulation. Ann. Rev. Physiol. 34:13-46, 1972.

10. White, R. J. Summary Report on a Basic Model of Circulatory, Fluid, and Electrolyte Regulation in the Human System Based upon the Model of Guyton, NASA CR-160212, 1973

11. Altchuler, S. I., Brand, S. N., and White, R. J. A mathematical model of calcium metabolism. Preprints of the 1981 Annual Scientific Meeting of the Aerospace Medical Association, 1981, pp. 309-310.

12. Fitzjerrell, D. G., Grounds, D. J., and Leonard, J. I. Study Report on Interfacing Major Physiological Subsystem Models: An Approach for Developing a Whole-Body Algorithm, NASA CR-160232, 1975.

13. White, R. J. Long-term regulation in the cardiovascular system: Cornerstone in the development of a composite physiological model. Modeling and Simulation 5:477-482, 1974.

14. White, R. J., and Croston, R.C. Human physiological problems in zero gravity: An attempt at understanding through systems analysis. Proc. 1974 SCSC, Simulation Councils, La Jolla, 743-747, 1974

15. Leonard, J. I., Rummel, J. A., and Croston, R. C. Hypothesis testing of physiological adaptation to zero gravity using simulation models. Proc. 28th ACEMB 17, 1975.

16. Leonard, J. I., Leach, C. S., and Rummel, J. A. Computer simulations of postural change, water immersion, and bed rest: An integrated approach for understanding the space-flight response. Physiologist 22:5-31 to $5-32,1979$

17. Leonard, J. I., White, R. J., and Rummel, J. A. An intergrative approach to space-flight physiology using systems analysis and mathematical simulation. Proc. 11th Space Simulation Conf., NASA Conference Publication 2150,149-162, 1980.

18. Blomqvist, C. G., Nixon, J. V., Johnson, R. L., and Mitchell, J. H. Early cardiovascular adaptation to zero gravity simulated by head-down tilt. Acta Astronautica 7:543-553, 1980.

19. Gauer, O. H. Recent advances in the physiology of whole body immersion. Acta Astronautica 2:31-39, 1975.

20. Epstein, M. Renal effects of headout water immersion in man: Implications for an understanding of volume homeostasis. Physiol Rev. 58:529-581, 1978.

21. Johnston, R. S., Dietlein, L. F., and Berry, C. A., eds., Biomedical Results of Apollo, NASA SP-368, 1975.

22. Johnston, R. S., and Dietlein, L. F., eds., Biomedical Results From Skylab, NASA SP-377, 1977.

23. Anderson, M. Biospex: Biological Space Experiments, A Compendium of Life Sciences Experiments Carried on U.S. Spacecraft, NASA TM-58217, 1979.

24. Buderer, M. D. Russian Biospex: Biological Space Experiments, A Space Life Sciences Bibliography, NASA JSC-17072, 1981 